\title{
Fréchet Envelopes of Nonlocally Convex Variable Exponent Hörmander Spaces
}

\author{
Joaquín Motos, María Jesús Planells, and César F. Talavera \\ Departamento de Matemática Aplicada, Universidad Politécnica de Valencia, Camino de Vera, s/n, 46022 Valencia, Spain \\ Correspondence should be addressed to Joaquín Motos; jmotos@mat.upv.es
}

Received 30 May 2016; Accepted 27 September 2016

Academic Editor: Hatem Mejjaoli

Copyright (C) 2016 Joaquín Motos et al. This is an open access article distributed under the Creative Commons Attribution License, which permits unrestricted use, distribution, and reproduction in any medium, provided the original work is properly cited.

\begin{abstract}
We show that the dual $\left(B_{p(\cdot)}^{\text {loc }}(\Omega)\right)^{\prime}$ of the variable exponent Hörmander space $B_{p(\cdot)}^{\text {loc }}(\Omega)$ is isomorphic to the Hörmander space $B_{\infty}^{c}(\Omega)$ (when the exponent $p(\cdot)$ satisfies the conditions $0<p^{-} \leq p^{+} \leq 1$, the Hardy-Littlewood maximal operator $M$ is bounded on $L_{p(\cdot) / p_{0}}$ for some $0<p_{0}<p^{-}$and $\Omega$ is an open set in $\left.\mathbb{R}^{n}\right)$ and that the Fréchet envelope of $B_{p(\cdot)}^{\text {loc }}(\Omega)$ is the space $B_{1}^{\text {loc }}(\Omega)$. Our proofs rely heavily on the properties of the Banach envelopes of the $p_{0}$-Banach local spaces of $B_{p(\cdot)}^{\text {loc }}(\Omega)$ and on the inequalities established in the extrapolation theorems in variable Lebesgue spaces of entire analytic functions obtained in a previous article. Other results for $p(\cdot) \equiv p, 0<p<1$, are also given (e.g., all quasi-Banach subspace of $B_{p}^{\text {loc }}(\Omega)$ is isomorphic to a subspace of $l_{p}$, or $l_{\infty}$ is not isomorphic to a complemented subspace of the Shapiro space $h_{p^{-}}$). Finally, some questions are proposed.
\end{abstract}

Dedicated to the memory of Nigel J. Kalton

\section{Introduction and Notation}

The Lebesgue spaces $L_{p(\cdot)}$ with variable exponent and the corresponding Sobolev spaces $W_{p(\cdot)}^{m}$ have been the subject of considerable interest since the early 1990s. These spaces are of interest in their own right and also have applications to PDEs of nonstandard growth and to modelling electrorheological fluids and to image restoration. For a thorough discussion of these spaces and their history, see $[1,2]$. Our paper lies in this field of variable exponent function spaces and is a continuation of [3] (see also $[4,5]$ ). In [5] the (nonweighted) variable exponent Hörmander spaces $B_{p(\cdot)}, B_{p(\cdot)}^{c}(\Omega)$, and $B_{p(\cdot)}^{\text {loc }}(\Omega)$ were introduced (recall that the classical Hörmander spaces $B_{p, k}$, $B_{p, k}^{c}(\Omega)$, and $B_{p, k}^{\text {loc }}(\Omega)$ play a crucial role in the theory of linear partial differential operators (see, e.g., [6-10])) and there, extending a Hörmander result $[6$, Chapter XV] to our context, the dual of $B_{p(\cdot)}^{c}(\Omega)$ (when $1<p^{-} \leq p^{+}<\infty$ ) was calculated (as a consequence some results on sequence space representation of variable exponent Hörmander spaces were obtained). In [3] the dual $\left(B_{p(\cdot)}^{c}(\Omega)\right)^{\prime}$ was calculated when $0<p^{-} \leq p^{+} \leq 1$ (with techniques necessarily different from those used in [5]) and a number of applications were given. In the current article we show that the dual $\left(B_{p(\cdot)}^{\text {loc }}(\Omega)\right)^{\prime}$ is isomorphic to $B_{\infty}^{c}(\Omega)$ (when $0<p^{-} \leq p^{+} \leq 1$ ) and that the Fréchet envelope of $B_{p(\cdot)}^{\text {loc }}(\Omega)$ is $B_{1}^{\text {loc }}(\Omega)$. Applications to the study of the structure of complemented subspaces of $B_{p(\cdot)}^{\text {loc }}(\Omega)$ are also given. The techniques used in the article (also in [3]) are based on the inequalities of the extrapolation theorems obtained by the authors in [4] and on the properties of the Banach envelopes of the $p_{0}$-Banach local spaces of $B_{p(\cdot)}^{\text {loc }}(\Omega)$. Finally, three questions on duality and on sequence space representation of variable exponent Hörmander spaces are proposed.

\subsection{Notation}

(1) Let $E$ and $F$ be Hausdorff topological linear spaces over $\mathbb{C}$. If $E$ and $F$ are isomorphic (i.e., there exists a linear homeomorphism from $E$ onto $F$ ) we put 
$E \simeq F$. The (topological) dual of $E$ is denoted by $E^{\prime}$ and is given (unless otherwise stated) the topology of uniform convergence on all the bounded subsets of $E$ (sometimes denoted by $\beta\left(E^{\prime}, E\right)$ ). The completion of $E$ is denoted by $\widetilde{E}$. If $E$ is metrizable and complete, $E$ is said to be an $F$-space. A locally convex $F$-space is said to be a Fréchet space. We put $E \hookrightarrow F$ if $E$ is a linear subspace of $F$ and the canonical injection is continuous. If $E$ is a Banach space, $E^{\mathbb{N}}$ (resp., $E^{(\mathbb{N})}$ ) is the topological product (resp., the locally convex direct sum) of a countable number of copies of $E . \mathbb{C}^{\mathbb{N}}$ (resp., $\mathbb{C}^{(\mathbb{N})}$ ) is denoted by $\omega$ (resp., $\varphi$ ). For unexplained notation we refer to [11-14].

(2) If $f \in L_{1}\left(\mathbb{R}^{n}\right)$ the Fourier transform of $f, \hat{f}$ or $\mathscr{F} f$, is defined by $\widehat{f}(\xi)=\int_{\mathbb{R}^{n}} f(x) e^{-i \xi x} d x$. If $f$ is a function on $\mathbb{R}^{n}$, then $\tilde{f}(x)=f(-x)$ for $x \in \mathbb{R}^{n} . B_{r}$ is the closed Euclidean ball $\{x:|x| \leq r\}$ in $\mathbb{R}^{n} . C_{0}^{\infty}\left(\mathbb{R}^{n}\right), C_{0}^{\infty}(\Omega)$, and $S\left(\mathbb{R}^{n}\right)$ are the usual Schwartz spaces (in the last space the norms $\max _{|\alpha| \leq m} \sup _{x \in \mathbb{R}^{n}}\left(1+|x|^{2}\right)^{m}\left|\partial^{\alpha} \varphi(x)\right|$, $m=0,1,2, \ldots$, are denoted by $\left.|\varphi|_{m}\right) . D^{\prime}\left(\mathbb{R}^{n}\right), D^{\prime}(\Omega)$, and $S^{\prime}\left(\mathbb{R}^{n}\right)$ are their corresponding duals. $\mathscr{E}^{\prime}(K)(K$ compact in $\mathbb{R}^{n}$ ) is the set of distributions on $\mathbb{R}^{n}$ with support contained in $K$. The Fourier transform in $S^{\prime}\left(\mathbb{R}^{n}\right)$ is also denoted by ${ }^{\wedge}$ (or $\left.\mathscr{F}\right)$. If $u \in S^{\prime}\left(\mathbb{R}^{n}\right)$, $\tilde{u}$ is defined by $\langle\varphi, \widetilde{u}\rangle=\langle\widetilde{\varphi}, u\rangle$ for all $\varphi \in S\left(\mathbb{R}^{n}\right)$; thus $\sim$ coincides with the operator $(2 \pi)^{-n} \mathscr{F}^{2}$. When we consider function spaces (or distribution spaces) defined on the whole Euclidean space $\mathbb{R}^{n}$, we shall omit the " $\mathbb{R}^{n \text { " }}$ of their notation. The letter $C$ will always denote a positive constant, not necessarily the same at each occurrence.

(3) Throughout this paper all vector spaces are assumed complex. By definition, a quasi-normed space is a vector space $X$ with a quasi-norm $x \rightarrow\|x\|$ satisfying (i) $\|x\|>0, x \neq 0$, (ii) $\|\alpha x\|=|\alpha|\|x\|$, and (iii) $\|x+y\| \leq C(\|x\|+\|y\|), x, y \in X$, for some $C$ independent of $x, y$. If $X$ is complete, we say it is a quasi-Banach space. The quasi-norm is $p$-subadditive for some $p>0$ if $\|x+y\|^{p} \leq\|x\|^{p}+\|y\|^{p}, x, y \in X$; in this case, if $X$ is complete, we say it is a $p$-Banach space. Recall that if a quasi-normed space $(X,\|\cdot\|)$ is locally convex then it becomes a normed space: Let $B_{X}=\{x:\|x\|<1\}$ be and let $U$ be a balanced convex open neighborhood of 0 such that $U \subset B_{X}$. If $\epsilon>0$ is such that $\epsilon B_{X} \subset U$ then the Minkowski functional of $U,\|\cdot\|_{U}\left(\|\cdot\|_{U}=\inf \{\lambda>0: x \in \lambda U\}\right)$, is a norm equivalent to $\|\cdot\|$ since

$$
\epsilon\|x\|_{U} \leq\|x\| \leq\|x\|_{U}
$$

holds for all $x \in X$. (See [12, Chapter 1] and [15, Chapter 25].)

(4) $\mathscr{P}^{0}$ is the set of all measurable functions $p(\cdot)$ on $\mathbb{R}^{n}$ with range in $(0, \infty)$ such that $p^{-}=\operatorname{ess~inf}_{x \in \mathbb{R}^{n}} p(x)>$ 0 and $p^{+}=$ess $\sup _{x \in \mathbb{R}^{n}} p(x)<\infty$. $L_{p(\cdot)}$ denotes the set of all complex-valued measurable functions on $\mathbb{R}^{n}$ such that, for some $\lambda>0, \int_{\mathbb{R}^{n}}(|f(x)| / \lambda)^{p(x)} d x<\infty$. With the norm (quasi-norm if $p^{-}<1$ ) defined by $\|f\|_{p(\cdot)}:=\inf \left\{\lambda>0: \int_{\mathbb{R}^{n}}(|f(x)| / \lambda)^{p(x)} d x \leq 1\right\}, L_{p(\cdot)}$ becomes a Banach (quasi-Banach if $p^{-}<1$ ) space. If $p^{-}<1$ we can also define $L_{p(\cdot)}$ as the set of all measurable functions $f$ such that $|f|^{p_{0}} \in L_{q(\cdot)}$, where $0<p_{0} \leq p^{-}$and $q(x)=p(x) / p_{0}$. In this case we have $\|f\|_{p(\cdot)}=\left\||f|^{p_{0}}\right\|_{q(\cdot)}^{1 / p_{0}}$. (See $\left.[1,2,16].\right)$

(5) If $K$ is a compact subset of $\mathbb{R}^{n}$ and $0<p \leq \infty$, then $L_{p}^{K}:=\left\{f \in S^{\prime}: \operatorname{supp} \widehat{f} \subset K, f \in L_{p}\right\} .\left(L_{p}^{K},\|\cdot\|_{p}\right)$ is a quasi-Banach (Banach if $p \geq 1$ ) space (see [17, Chapters 1,2$])$. If $p(\cdot) \in \mathscr{P}^{0}$ then

$L_{p(\cdot)}^{K}:=\left\{f \in S^{\prime}: \operatorname{supp} \hat{f} \subset K,\|f\|_{p(\cdot)}<\infty\right\}$.

$\left(L_{p(\cdot)}^{K},\|\cdot\|_{p(\cdot)}\right)$ is a quasi-normed space (normed if $p^{-} \geq$ 1) linear space. From the Paley-Wiener-Schwartz theorem it follows that the elements of $L_{p(\cdot)}^{K}$ are entire analytic functions of exponential type. When $p(\cdot) \equiv$ $p$, a constant, then $L_{p(\cdot)}^{K}=L_{p}^{K}$ with equality of quasinorms (resp., norms). We shall denote by $S^{K}$ the collection of all $f \in S$ such that supp $\hat{f} \subset K$; obviously $S^{K} \subset L_{p(\cdot)}^{K}$. The spaces $L_{p(\cdot)}^{K}$ have been introduced and studied in [4].

(6) Let $p(\cdot) \in \mathscr{P}^{0}$ be and let $\Omega$ be an open set in $\mathbb{R}^{n}$. Then

$$
\begin{gathered}
B_{p(\cdot)}:=\left\{u \in S^{\prime}: \widehat{u} \in L_{p(\cdot)}\left(\Longleftrightarrow \exists g \in L_{p(\cdot)}\right.\right. \\
\left.\left.\cap L_{1}^{\mathrm{loc}}:\langle\varphi, \widehat{u}\rangle=\int_{\mathbb{R}^{n}} \varphi g d x, \forall \varphi \in C_{0}^{\infty}\right)\right\} .
\end{gathered}
$$

If $u \in B_{p(\cdot)}$ we put $\|u\|_{B_{p(\cdot)}}:=\|\widehat{u}\|_{p(\cdot)} \cdot\left(B_{p(\cdot)},\|\cdot\|_{B_{p(\cdot)}}\right)$ is a quasi-normed space (a Banach space isomorphic to $L_{p(\cdot)}$ if $\left.p^{-} \geq 1\right)$. Now consider the space

$B_{p(\cdot)}^{c}(\Omega):=\bigcup\left\{B_{p(\cdot)} \cap \mathscr{E}^{\prime}(K): K\right.$ compact in $\left.\Omega\right\}$.

If every $B_{p(\cdot)} \cap \mathscr{E}^{\prime}(K)$ is equipped with the topology induced by $B_{p(\cdot)}$, then $B_{p(\cdot)}^{c}(\Omega)$ (endowed with the corresponding inductive linear topology) becomes a strict inductive limit

$$
B_{p(\cdot)}^{c}(\Omega):=\operatorname{ind}_{K}\left[B_{p(\cdot)} \cap \mathscr{E}^{\prime}(K)\right] .
$$

(Each step $B_{p(\cdot)} \cap \mathscr{E}^{\prime}(K)$ is a quasi-Banach space since it is isomorphic to $L_{p(\cdot)}^{-K}$ via the Fourier transform and this space is a quasi-Banach space by [4, Theorem 3.5]. On the other hand, the bilinear mapping $S \times\left(B_{p} \cap \mathscr{E}^{\prime}(K)\right) \rightarrow B_{p} \cap \mathscr{E}^{\prime}(K):(\varphi, u) \rightarrow \varphi u$ is continuous (see [5])). Finally,

$$
B_{p(\cdot)}^{\mathrm{loc}}(\Omega):=\left\{u \in D^{\prime}(\Omega): \varphi u \in B_{p(\cdot)}, \forall \varphi \in C_{0}^{\infty}(\Omega)\right\} .
$$


The topology of this space is generated by the seminorms $\left(p_{0}\right.$-seminorms when $p^{-}<1$; here $p_{0} \in$ $\left.\left(0, p^{-}\right)\right) u \rightarrow\|u\|_{p(\cdot), \varphi}:=\|\varphi u\|_{B_{p(\cdot)}}, \varphi \in C_{0}^{\infty}(\Omega)$.

The spaces $B_{p(\cdot)}, B_{p(\cdot)}^{c}(\Omega)$, and $B_{p(\cdot)}^{\text {loc }}(\Omega)$ are called variable exponent Hörmander spaces and have been introduced in [5]. If $p(\cdot) \equiv p$ and $p \geq 1$, these spaces coincide with the Hörmander spaces $B_{p, 1}, B_{p, 1}^{\text {loc }}(\Omega)$, and $B_{p, 1}^{\text {loc }}(\Omega)$, respectively (see [6]). Throughout this paper, $B_{\infty}^{\text {loc }}(\Omega)$ will denote the Hörmander space $B_{\infty, 1}^{\text {loc }}(\Omega)$ (see again $[6$, Chapter $\mathrm{X}]$ ).

(7) We conclude this section recalling some basic facts about the Banach envelope of a quasi-normed space and the Fréchet envelope of a metrizable topological linear space.

Let $\left(X,\|\cdot\|_{X}\right)$ be a quasi-normed space whose dual $X^{\prime}$ separates the points of $X$ and let $B_{X}$ be the unit ball of $X$. Then $X^{\prime}$ is a Banach space under the norm $\left\|x^{\prime}\right\|=\sup \left\{\left|\left\langle x, x^{\prime}\right\rangle\right|: x \in B_{X}\right\}$. The Banach envelope $\widehat{X}$ of $\left(X,\|\cdot\|_{X}\right)$ is the completion of $X$ in the norm $\|\cdot\|_{c}$ defined by

$$
\|x\|_{c}:=\sup \left\{\left|\left\langle x, x^{\prime}\right\rangle\right|:\left\|x^{\prime}\right\| \leq 1\right\} .
$$

$\|\cdot\|_{c}$ coincides with the Minkowski functional of the convex hull of $B_{X},\|\cdot\|_{c} \leq\|\cdot\|_{X}$ and the inclusion $X \hookrightarrow \widehat{X}$ is continuous with dense range (if $X$ is a Banach space then $X=\widehat{X}) . \widehat{X}$ has the property that any bounded linear operator $L: X \rightarrow Y$ into a Banach space extends with preservation of norm to a bounded linear operator $\widehat{L}: \widehat{X} \rightarrow Y$; thus $(\widehat{X})^{\prime}$ (and $\left.\left(X,\|\cdot\|_{c}\right)^{\prime}\right)$ becomes linearly isometric to $X^{\prime}$ (see, e.g., [12, pp. 27, $28]$ and [18, Section 2]; in the last paper the Banach envelopes of some Besov and Triebel-Lizorkin spaces are computed; in [19] the Banach envelope of PaleyWiener type spaces is also computed).

Now let $X[\mathscr{T}]$ be a metrizable topological linear space such that its dual $X^{\prime}\left(=(X[\mathscr{T}])^{\prime}\right)$ separates points of $X$. The Mackey topology of $X[\mathscr{T}], m\left(X, X^{\prime}\right)$, is the finest locally convex topology on $X$ which has $X^{\prime}$ as dual space. If $\left\{U_{n}\right\}_{n=1}^{\infty}$ is a base of balanced neighborhoods of zero for $\mathscr{T}$ then $\left\{\widetilde{U}_{n}\right\}_{n=1}^{\infty}$, where $\widetilde{U}_{n}$ denotes the $\mathscr{T}$-closed convex hull of $U_{n}$, is a base of neighborhoods of zero for $m\left(X, X^{\prime}\right)$ and thus this topology is metrizable. The Fréchet envelope $\widehat{X}$ of $X[\mathscr{T}]$ is the completion of $X\left[m\left(X, X^{\prime}\right)\right](\widehat{X}=X[\mathscr{T}]$ when $X[\mathscr{T}]$ is a Fréchet space). $\widehat{X}$ coincides with the Banach envelope of $X[\mathscr{T}]$ when this space is quasinormed. If $j$ is the canonical injection of $X[\mathscr{T}]$ into $\widehat{X}$, then the transpose of $j$ is an algebraic isomorphism of $(\widehat{X})^{\prime}$ onto $(X[\mathscr{T}])^{\prime}$. If $X$ and $Y$ are metrizable topological linear spaces with separating duals and $T$ is a continuous linear mapping taking $X$ into $Y$, then $T$ is also continuous from $X\left[m\left(X, X^{\prime}\right)\right]$ into $Y\left[m\left(Y, Y^{\prime}\right)\right]$ and so there is a unique extension $\widehat{T}$ of $T$ to a continuous linear mapping taking $\widehat{X}$ into $\widehat{Y}$.
If in addition $X$ and $Y$ are $F$-spaces and $T(X)=Y$, then $\widehat{T}(\widehat{X})=\widehat{Y}$. (See the proofs of these results in $[20,21]$; furthermore, in these papers and in [12], the Fréchet envelopes of several $F$-spaces of holomorphic and harmonic functions are computed.)

\section{The Dual and the Fréchet Envelope of $B_{p(\cdot)}^{\text {loc }}(\Omega)\left(0<p^{-} \leq p^{+} \leq 1\right)$}

In [6], the isomorphism $\left(B_{2, k}^{c}(\Omega)\right)^{\prime} \simeq B_{2,1 / \tilde{k}}^{\text {loc }}(\Omega)$ is shown (being $\Omega$ an open convex set in $\mathbb{R}^{n}$ and $k$ a weight satisfying the estimate $k(x+y) \leq(1+C|x|)^{N} k(y), x, y \in \mathbb{R}^{n}, C$ and $N$ positive constants). In Theorem 4.3 of [5] this isomorphism is extended to variable exponent Hörmander spaces with $1<$ $p^{-} \leq p^{+}<\infty:\left(B_{p(\cdot)}^{c}(\Omega)\right)^{\prime} \simeq B \frac{\text { loc }}{p^{\prime}(\cdot)}(\Omega)$. In [3] it is shown that $\left(B_{p(\cdot)}^{c}(\Omega)\right)^{\prime} \simeq B_{\infty}^{\text {loc }}(\Omega)$ when the exponent $p(\cdot)$ satisfies $0<p^{-} \leq p^{+} \leq 1$ (the techniques used are different from those used in [5] since if $p^{+}<1$ the dual of $L_{p(\cdot)}$ is trivial and the steps $B_{p(\cdot)} \cap \mathscr{E}^{\prime}(K)$ are quasi-Banach spaces instead of Banach spaces) and several applications of this result were given.

As a consequence [5, Theorem 4.3] and the reflexivity of $L_{p(\cdot)}$ (see [1, Corollary 2.81]) one gets the isomorphism $\left(B_{p(\cdot)}^{\text {loc }}(\Omega)\right)^{\prime} \simeq B_{p^{\prime}(\cdot)}^{c}(\Omega)$ when $1<p^{-} \leq p^{+}<\infty$ and the Hardy-Littlewood maximal operator is bounded on $L_{p(\cdot)}$ and $L_{\widetilde{p(\cdot)}}$. In this section we show the $p^{+} \leq 1$ counterpart of this result: the dual $\left(B_{p(\cdot)}^{\text {loc }}(\Omega)\right)^{\prime}$ (equipped with the topology $\mathfrak{I}$ of the uniform convergence on $m\left(B_{p(\cdot)}^{\text {loc }}(\Omega),\left(B_{p(\cdot)}^{\text {loc }}(\Omega)\right)^{\prime}\right)$ bounded subsets of $B_{p(\cdot)}^{\text {loc }}(\Omega)$ ) is isomorphic to $B_{\infty}^{c}(\Omega)$ (and therefore to $l_{\infty}^{(\mathbb{N})}$ ) when $0<p^{-} \leq p^{+} \leq 1$ and the HardyLittlewood maximal operator $M$ is bounded on $L_{p(\cdot) / p_{0}}$. Our proof is based on the inequalities obtained in the extrapolation theorem [4, Theorem 3.5], on the properties of the Banach envelopes of the $p_{0}$-Banach local spaces of $B_{p(\cdot)}^{\text {loc }}(\Omega)$, and on the identification of the Fréchet envelope of $B_{p(\cdot)}^{\text {loc }}(\Omega)$. We also give a characterization of the locally convex complemented subspaces of $B_{p(\cdot)}^{\text {loc }}(\Omega)$ and we show that $l_{\infty}$ is not isomorphic to a complemented subspace of the Shapiro space $h_{p^{-}}$(see Remark 8(1) to Theorem 7). Note that Theorem 7 can have independent interest to calculate Fréchet envelopes of $F$-spaces.

Throughout the entire article, $p(\cdot)$ denotes a variable exponent in $\mathscr{P}^{0}$ such that $0<p^{-} \leq p^{+} \leq 1$ and the HardyLittlewood maximal operator $M$ is bounded on $L_{p(\cdot) / p_{0}}$ for some $0<p_{0}<p^{-}, \Omega$ denotes an open set in $\mathbb{R}^{n},\left\{K_{j}\right\}_{j=1}^{\infty}$ is a fundamental sequence of compact subsets of $\Omega$ such that, for all $j, K_{j}=\bar{\circ}_{\stackrel{\circ}{K}_{j}}$ and $\stackrel{\circ}{K}_{j}$ has the segment property, and $\left\{\theta_{j}\right\}_{j=1}^{\infty}$ is a $C_{0}^{\infty}(\Omega)$-partition of unity on $\Omega$ such that $\operatorname{supp} \theta_{j} \subset K_{j}$ for every $j$. Finally, $\left\{\chi_{j}\right\}_{j=1}^{\infty}$ denotes a sequence in $C_{0}^{\infty}(\Omega)$ such that $\chi_{j} \equiv 1$ on $K_{j}$ and supp $\chi_{j} \subset \stackrel{\circ}{K}_{j+1}$ for each $j$. 
Recall (see Section 1 and [5]) that $B_{p(\cdot)}^{\text {loc }}(\Omega)$, with the topology defined by the collection of $p_{0}$-seminorms $\left\{\|\cdot\|_{p(\cdot), \chi_{j}}: j=\right.$ $1,2, \ldots\}$, becomes an $F$-space (actually, a locally $p_{0}$-convex space) and that $\|\cdot\|_{p(\cdot), \chi_{j}} \leq C_{j}\|\cdot\|_{p(\cdot), \chi_{j+1}}$ holds for all $j$. The family $\left\{V_{j, \varepsilon}: j \in \mathbb{N}, \varepsilon>0\right\}$, where $V_{j, \varepsilon}=\left\{u \in B_{p(\cdot)}^{\text {loc }}(\Omega)\right.$ : $\left.\|u\|_{p(\cdot), \chi_{j}}<\varepsilon\right\}$, is a base of neighborhoods of 0 in $B_{p(\cdot)}^{\operatorname{loc}}(\Omega)$.

Lemma 1. $X:=\left(B_{p(\cdot)}^{\mathrm{loc}}(\Omega) / \operatorname{ker}\|\cdot\|_{p(\cdot), \chi_{j}},\|\cdot\|_{p(\cdot), \chi_{j}}^{*}\right)$ is an infinite dimensional $p_{0}$-normed space whose dual separates points of $X$ (here $\|\cdot\|_{p(\cdot), \chi}^{*}$ is the corresponding quotient $p_{0}$-norm). If $p(\cdot) \equiv$ $p, 0<p<1$, then $X$ becomes an infinite dimensional $p$ normed space with separating dual.

Proof. If $u \in B_{p(\cdot)}^{\text {loc }}(\Omega),[u]_{j}$ denotes the coset of $u$. Then $\left\{[\varphi]_{j}: \varphi \in C_{0}^{\infty}\left(K_{j}\right)\right\}$ is an infinite dimensional subspace of $B_{p(\cdot)}^{\text {loc }}(\Omega) / \operatorname{ker}\|\cdot\|_{p(\cdot), \chi_{j}}$ (see also [5, Theorem 3.7/2]). Now, for each $\varphi \in S$, put $\left\langle[u]_{j}, U \varphi\right\rangle:=\left\langle\varphi, \chi_{j} u\right\rangle$. Let us see that $U \varphi \in X^{\prime}$. Naturally, $U \varphi$ is well defined (if $v \in[u]_{j}$ then $\left.\chi_{j} \nu=\chi_{j} u\right)$. Furthermore, of the embedding $L_{p(\cdot)}^{-K_{j+1}} \hookrightarrow L_{1}^{-K_{j+1}}$ (see [4, Theorem 3.5/5]) and the fact that for $u \in B_{p(\cdot)}^{\text {loc }}(\Omega)$ one has $\chi_{j} u \in B_{p(\cdot)} \cap \mathscr{E}^{\prime}\left(K_{j+1}\right)$, that is, $\left(\chi_{j} u\right)^{\wedge} \in L_{p(\cdot)}^{-K_{j+1}}$, it follows that

$$
\begin{aligned}
\left\langle[u]_{j}, U \varphi\right\rangle & =\left\langle\varphi, \chi_{j} u\right\rangle=(2 \pi)^{-n}\left\langle\widehat{\tilde{\varphi}},\left(\chi_{j} u\right)^{\wedge}\right\rangle \\
& =(2 \pi)^{-n} \int_{\mathbb{R}^{n}} \widehat{\widetilde{\varphi}}\left(\chi_{j} u\right)^{\wedge} d x, \\
\left|\left\langle[u]_{j}, U \varphi\right\rangle\right| & \leq(2 \pi)^{-n} \int_{\mathbb{R}^{n}}|\widehat{\widetilde{\varphi}}|\left|\left(\chi_{j} u\right)^{\wedge}\right| d x \\
& \leq(2 \pi)^{-n}\|\hat{\varphi}\|_{\infty}\left\|\left(\chi_{j} u\right)^{\wedge}\right\|_{1} \\
& \leq C\|\hat{\varphi}\|_{\infty}\left\|\left(\chi_{j} u\right)^{\wedge}\right\|_{p(\cdot)} \\
& =C\|\hat{\varphi}\|_{\infty}\|u\|_{p(\cdot), \chi_{j}} \\
& =\left(C\|\hat{\varphi}\|_{\infty}\right)\left\|[u]_{j}\right\|_{p(\cdot), \chi_{j}}^{*},
\end{aligned}
$$

which proves that $U \varphi \in X^{\prime}$. Hence the required conclusion follows easily.

The second part of lemma is obvious taking into account that $\|\cdot\|_{p, \chi_{j}}^{*}$ is a $p$-norm and that [5, Theorem 3.7/2] and [4, Theorem 3.5/5] are also valid when $p(\cdot) \equiv p$ because the Hardy-Littlewood maximal operator is bounded in $L_{p / p_{0}}$ for all $0<p_{0}<p$.

Remark 2. Naturally in the second part of the previous lemma we could apply [17, Proposition 1.3.2, p. 17] instead of [4, Theorem 3.5/5].

Lemma 3. Let $E[\mathfrak{T}]$ be a locally $p$-convex space $(0<p<1)$ and metrizable whose topology is defined by a family of $p$ seminorms $\left\{\|\cdot\|_{n}: n \geq 1\right\}$ such that, for every $m<n,\|\cdot\|_{m} \leq$ $C_{m, n}\|\cdot\|_{n}\left(C_{m, n}\right.$ constants $\left.>0\right)$. Let $Q$ be a (complemented) quasi-normed subspace of E. Then there exists $k$ such that, for each $r \geq k, Q$ is isomorphic to a (complemented) subspace of the local p-normed space $E_{r}=\left(E / \operatorname{ker}\|\cdot\|_{r},\|\cdot\|_{r}^{*}\right)$. If furthermore $Q$ is complete, that is, a quasi-Banach space, then $Q$ is isomorphic to a (complemented) subspace of the local pBanach space $\widetilde{E_{r}}$.

Proof. Let $\|\cdot\|_{Q}$ be the quasi-norm on $Q$ which generates the topology of $Q$. Then the identity $\mathrm{id}_{Q}:\left(Q,\|\cdot\|_{Q}\right) \rightarrow Q[\mathfrak{I}]$ is an isomorphism. Thus, for every $n$, there exists an $M_{n}>0$ such that $\|x\|_{n} \leq M_{n}\|x\|_{\mathrm{Q}}$ for all $x \in Q$, and there exist also an integer $m$ and $C>0$ so that $\|x\|_{Q} \leq C\|x\|_{m}$ for all $x \in Q$. Next, fix $n \geq m$. Then, for every $x \in Q$, we have

$$
\|x\|_{n} \leq M_{n}\|x\|_{\mathrm{Q}} \leq M_{n} C\|x\|_{m} \leq M_{n} C C_{m, n}\|x\|_{n},
$$

which shows that on $Q\|\cdot\|_{n}$ is a $p$-norm equivalent to $\|\cdot\|_{Q}$. Furthermore, these inequalities prove immediately that the restriction to $Q$ of the canonical mapping $\pi_{n}: E \rightarrow E_{n}: x \rightarrow$ $[x]_{n}$ is an isomorphism onto $\pi_{n}(Q)$.

If $Q$ is complemented in $E$ and $P$ is a continuous projection in $E$ such that $\operatorname{Im} P=Q$, there exist an integer $k \geq m$ and a constant $B>0$ such that $\|P x\|_{m} \leq B\|x\|_{k}$ for every $x \in E$. Then it is easy to check that, for every $r \geq k$, the mapping $P_{r}: E_{r} \rightarrow E_{r}$ defined by $P_{r}\left([x]_{r}\right)=[P x]_{r}$ is a continuous projection such that $\operatorname{Im} P_{r}=\pi_{r}(Q)$.

Finally, if $Q$ is complete then the extension of $P_{r}$ to $\widetilde{E_{r}}, \widetilde{P_{r}}$, is a continuous projection in $\widetilde{E_{r}}$ such that $\operatorname{Im} \widetilde{P_{r}}=\pi_{r}(Q)$.

Remark 4. This lemma is well known in the locally convex case (see, e.g., [22]).

Proposition 5. Let $p(\cdot) \equiv p, 0<p<1$, and $X:=$ $\left(B_{p}^{\text {loc }}(\Omega) / \operatorname{ker}\|\cdot\|_{p, \chi_{j}},\|\cdot\|_{p, \chi_{j}}^{*}\right)$. Then, consider the following:

(1) The completion of $X$ is a p-Banach space ( $\infty$-dimensional and with separating dual) isomorphic to a subspace of $l_{p}$ and contains a subspace isomorphic to $l_{p}$.

(2) $B_{p}^{\text {loc }}(\Omega)$ is not locally convex.

(3) If $0<p<q \leq 1$, then $B^{\text {loc }}(\Omega) \varsubsetneqq B_{q}^{\text {loc }}(\Omega)$.

(4) All quasi-Banach subspace of $B_{p}^{\mathrm{loc}}(\Omega)$ is isomorphic to a subspace of $l_{p}$.

Proof. (1) Since the operator $X \rightarrow\left\{\chi_{j} u: u \in B_{p(\cdot)}^{\text {loc }}(\Omega)\right\}(c$ $\left.B_{p} \cap \mathscr{E}^{\prime}\left(K_{j+1}\right)\right):[u]_{j} \rightarrow \chi_{j} u$ is an isometry, the completion of $X$ is a $p$-Banach space isometric to a closed subspace of $B_{p} \cap \mathscr{E}^{\prime}\left(K_{j+1}\right)$. Let $a, b>0$ be such that $b<\pi$ and $K_{j+1} \subset$ $[-a, a]^{n}$ and consider the following diagram:

$$
\begin{aligned}
B_{p} \cap \mathscr{E}^{\prime}\left(K_{j+1}\right) & \stackrel{\wedge}{\longrightarrow} L_{p}^{-K_{j+1}} \stackrel{j}{\longrightarrow} L_{p}^{[-a, a]^{n}} \stackrel{s}{\longrightarrow} L_{p}^{[-b, b]^{n}} \\
& \stackrel{D}{\longrightarrow} l_{p}\left(\mathbb{Z}^{n}\right) \simeq l_{p},
\end{aligned}
$$

where $\wedge$ is the Fourier transform, $j$ is the canonical injection, $s$ is the isomorphism defined by $s(f)=f((b / a) \cdot)$, and $D$ is 
the isomorphic embedding defined by $D(f)=(f(k))_{k \in \mathbb{Z}^{n}}$ (this property of $D$ is well known; see, e.g., [23, pp. 101, 197] for $n=1$ and [24, Lemma 1.8, p. 17] for $n \geq 1$ ). The proof concludes composing these operators with the former isometric isomorphism. The second claim is a consequence of a result of Stiles (see, e.g., [12, Theorem 2.5]).

(2) First we observe that, for each compact $K \subset \Omega$, the restriction mapping $\Phi_{K}: B_{p} \cap \mathscr{E}^{\prime}(K) \rightarrow B_{p}^{\text {loc }}(\Omega): u \rightarrow$ $u \circ j \circ j_{\Omega}$ (here $j$ is the natural injection from $C_{0}^{\infty}$ into $S$ and $j_{\Omega}$ is the natural extension from $C_{0}^{\infty}(\Omega)$ into $C_{0}^{\infty}$ ) is continuous: If $u_{v} \rightarrow 0$ in $B_{p} \cap \mathscr{E}^{\prime}(K)$ then

$$
\begin{aligned}
\left\|\Phi_{K}\left(u_{\nu}\right)\right\|_{p, \varphi} & =\left\|\varphi \Phi_{K}\left(u_{\nu}\right)\right\|_{B_{p}}=\left\|\varphi u_{\nu}\right\|_{B_{p}} \\
& \leq C|\varphi|_{m}\left\|u_{\nu}\right\|_{B_{p}} \longrightarrow 0,
\end{aligned}
$$

for every $\varphi \in C_{0}^{\infty}(\Omega)$ (we have used the continuity of the bilinear mapping $S \times\left(B_{p} \cap \mathscr{E}^{\prime}(K)\right) \rightarrow B_{p} \cap \mathscr{E}^{\prime}(K):(\varphi, u) \rightarrow$ $\varphi u$; see (6) in Section 1.1). Next we show that the space $B_{p}^{\text {loc }}(\Omega)$ is not locally convex. Suppose otherwise and recall that the family $\left\{V_{j, \varepsilon}: j \in \mathbb{N}, \varepsilon>0\right\}$ is a local base of $B_{p}^{\text {loc }}(\Omega)$. Then, given $V_{j, \varepsilon}$ there exist an absolutely convex neighborhood $U$ of 0 and $V_{k, \delta}$ with $k>j$ such that $V_{j, \varepsilon} \supset U \supset V_{k, \delta}$ and so we have that $\varepsilon^{-1}\|u\|_{p, \chi_{j}} \leq p_{U}(u) \leq \delta^{-1}\|u\|_{p, \chi_{k}}$ holds for all $u \in B_{p}^{\text {loc }}(\Omega)$ ( $p_{U}$ is the Minkowski functional of $U$ ). We consider now the following commutative diagram

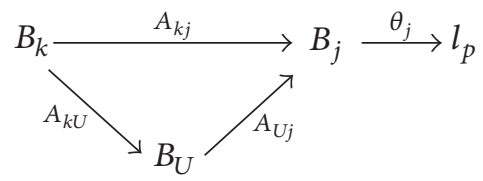

where $B_{k}$ (resp., $B_{j}$ ) is the completion of the $p$-normed space $\left(B_{p}^{\text {loc }}(\Omega) / \operatorname{ker}\|\cdot\|_{p, \chi_{k}},\|\cdot\|_{p, \chi_{k}}^{*}\right)\left(\operatorname{resp} .,\left(B_{p}^{\text {loc }}(\Omega) / \operatorname{ker} \|\right.\right.$. $\left.\left.\left\|_{p, \chi_{j}},\right\| \cdot \|_{p, \chi_{j}}^{*}\right)\right), B_{U}$ is the completion of the normed space $\left(B_{p}^{\text {loc }}(\Omega) / \operatorname{ker} p_{U}, p_{U}^{*}\right), A_{k j}$ (resp., $\left.A_{k U}, A_{U j}\right)$ denotes the extension of the natural operator $[u]_{k} \rightarrow[u]_{j}$ (resp., $[u]_{k} \rightarrow$ $[u]_{U},[u]_{U} \rightarrow[u]_{j}$ ), and $\theta_{j}$ is an isomorphism from $B_{j}$ onto $\operatorname{Im} \theta_{j}$ (see (1)). By a result of Stiles (see, e.g., [12, Proposition 2.9]), the operator $\theta_{j} \circ A_{U j}$ is compact but then, by the properties of $\theta_{j}, A_{U j}$ is also compact. From this and of $A_{k j}=A_{U j} \circ A_{k U}$, it follows that $A_{k j}$ is compact.

In order to complete the proof we consider a sequence $\left\{\varphi e^{i\left\langle\cdot, y_{v}\right\rangle}\right\}_{\nu=1}^{\infty}$ with $\varphi \in C_{0}^{\infty}\left(K_{j}\right) \backslash\{0\}$ and $y_{v} \rightarrow \infty$. Obviously, this sequence lies in $B_{p} \cap \mathscr{E}^{\prime}\left(K_{j}\right)$ and it is bounded here $\left(\left\|\varphi e^{i\left\langle\cdot, y_{\nu}\right\rangle}\right\|_{B_{p}}=\|\hat{\varphi}\|_{p}\right.$ for $\left.v=1,2, \ldots\right)$. Thus $\left\{\Phi_{K_{j}}\left(\varphi e^{i\left\langle\cdot, y_{\nu}\right\rangle}\right)\right\}_{\nu=1}^{\infty}=\left\{\varphi e^{i\left\langle\cdot, y_{\nu}\right\rangle}\right\}_{\nu=1}^{\infty}$ is bounded in $B_{p}^{\text {loc }}(\Omega)$ and so $\left\{\left[\varphi e^{i\left\langle\cdot, y_{\nu}\right\rangle}\right]_{k}\right\}_{\nu=1}^{\infty}$ is bounded in $B_{k}$ and since $A_{k j}$ is a compact operator we can find $\nu_{1}<v_{2}<\cdots$ such that the subsequence $\left\{\left[\varphi e^{i\left\langle\cdot, y_{\nu_{l}}\right\rangle}\right]_{k}\right\}_{l=1}^{\infty}$ converges in $B_{j}$. By applying (1), we see that $\chi_{j}\left(\varphi e^{i\left\langle\cdot, y_{v_{l}}\right\rangle}\right)=\varphi e^{i\left\langle\cdot y_{v_{l}}\right\rangle} \rightarrow \psi$ in $B_{p} \cap \mathscr{E}^{\prime}\left(K_{j+1}\right)$ but $\varphi e^{i\left\langle\cdot, y_{v_{l}}\right\rangle} \rightarrow 0$ in $S^{\prime}$ (because $y_{v_{l}} \rightarrow \infty$ ). Hence it follows that $\psi=0$, that is, that $\left\|\varphi e^{i\left\langle\cdot, y_{\nu}\right\rangle}\right\|_{B_{p}} \rightarrow 0$. This contradiction concludes the proof of (2).
(3) If $B_{p}^{\text {loc }}(\Omega)=B_{q}^{\text {loc }}(\Omega)$, then these spaces should be isomorphic by the open mapping theorem and so there exist positive integers $j \leq r \leq l$ and a constant $C>0$ such that $C^{-1}\|u\|_{p, \chi_{j}} \leq\|u\|_{q, \chi_{r}} \leq C\|u\|_{p, \chi_{l}}$ holds for all $u \in B_{p}^{\text {loc }}(\Omega)$. Hence and from the fact that $\chi_{i} \equiv 1$ on $K_{j}$ for every $i \geq j$, it follows that $C^{-1}\|\hat{\varphi}\|_{p}\left(=C^{-1}\left\|\left(\chi_{j} \varphi\right)^{\wedge}\right\|_{p}=C^{-1}\|\varphi\|_{p, \chi_{j}}\right) \leq$ $\|\hat{\varphi}\|_{q} \leq C\|\hat{\varphi}\|_{p}$ holds for all $\varphi \in C_{0}^{\infty}\left(K_{j}\right)$ and therefore that $C^{-1}\|\psi\|_{p} \leq\|\psi\|_{q} \leq C\|\psi\|_{p}$ is also valid for all $\psi \in$ $S^{-K_{j}}$. Then, by using the density of $S^{-K_{j}}$ in $L_{p}^{-K_{j}}$ (see [25, Proposition 1.4.4]) and the embedding $L_{p}^{-K_{j}} \hookrightarrow L_{q}^{-K_{j}}[17$, Proposition 1.3.2, p. 17], we get $C^{-1}\|f\|_{p} \leq\|f\|_{q} \leq C\|f\|_{p}$ for all $f \in L_{p}^{-K_{j}}$. This and the density of $S^{-K_{j}}$ in $L_{q}^{-K_{j}}$ imply that $L_{p}^{-K_{j}}=L_{q}^{-K_{j}}$ (coinciding algebraically and topologically). But then, reasoning as in the proof of (1), it is found that $l_{p}$ contains a subspace isomorphic to $l_{q}$ which contradicts a result of Stiles (see, e.g., [12, Corollary 2.8]).

(4) Let $Q$ be a quasi-Banach subspace of $B_{p}^{\text {loc }}(\Omega)$. By using Lemma 3, Q becomes isomorphic to a complemented subspace of the local $p$-Banach space $\left(B_{p}^{\text {loc }}(\Omega) / \operatorname{ker}\|\cdot\|_{p, \chi_{j}}\right.$, $\|\cdot\|_{p, \chi_{j}}^{*}$ ) for all large enough $j$. But we know by (1) that each of these spaces is isomorphic to a subspace of $l_{p}$. This concludes the proof of (4).

Theorem 6. $\left(B_{p(\cdot)}^{\text {loc }}(\Omega)\right)^{\prime}$ is algebraically isomorphic to $B_{\infty}^{c}(\Omega)$ when $0<p^{-} \leq p^{+} \leq 1$ (in particular $\left(B_{p}^{\mathrm{loc}}(\Omega)\right)^{\prime}$ is algebraically isomorphic to $B_{\infty}^{c}(\Omega)$ for all $\left.0<p \leq 1\right)$.

Proof. For each $j$ let $X_{j}$ be the normed space $\left(B_{p(\cdot)}^{\text {loc }}(\Omega) / \operatorname{ker} \|\right.$. $\left.\left\|_{p(\cdot), \chi_{j}},\right\| \cdot \|_{j}\right)$, where $\|\cdot\|_{j}$ is the Minkowski functional of the convex hull of the unit ball of the $p_{0}$-normed space $\left(B_{p(\cdot)}^{\text {loc }}(\Omega) / \operatorname{ker}\|\cdot\|_{p(\cdot), \chi_{j}},\|\cdot\|_{p(\cdot), \chi_{j}}^{*}\right)$. It is easily seen that the mapping $Z: B_{p(\cdot)}^{\text {loc }}(\Omega) \rightarrow \prod_{j=1}^{\infty} X_{j}$ is linear, injective, and continuous (for each $j$ one has $\left\|[u]_{j}\right\|_{j} \leq\left\|[u]_{j}\right\|_{p(\cdot), \chi_{j}}^{*}=$ $\|u\|_{p(\cdot), \chi_{j}}$ for all $u \in B_{p(\cdot)}^{\text {loc }}(\Omega)$, and so $\operatorname{pr}_{j} \circ Z$ is continuous $)$.

Let $L$ be a continuous linear functional on $B_{p(\cdot)}^{\text {loc }}(\Omega)$ and let $l$ and $C$ be such that $|\langle u, L\rangle| \leq C\|u\|_{p(\cdot), \chi_{l}}$ holds for all $u \in B_{p(\cdot)}^{\text {loc }}(\Omega)$. The linear functional $L_{l}: X_{l} \rightarrow \mathbb{C}$ defined by $\left\langle[u]_{l}, L_{l}\right\rangle=\langle u, L\rangle$ is continuous also since it is in the dual $\left(B_{p(\cdot)}^{\text {loc }}(\Omega) / \operatorname{ker}\|\cdot\|_{p(\cdot), \chi_{l}},\|\cdot\|_{p(\cdot), \chi_{l}}^{*}\right)^{\prime}$ and this space and $X_{l}^{\prime}$ are linearly isometric (Lemma 1 and (7), Section 1); therefore we get $\left|\left\langle[u]_{l}, L_{l}\right\rangle\right| \leq C\left\|[u]_{l}\right\|_{l}$. Hence it follows that the linear functional $L \circ Z^{-1}$ is continuous on $\operatorname{Im} Z$ : the family of seminorms $\left\{\left\|\left(x_{j}\right)\right\|_{(N)}:=\sum_{j=1}^{N}\left\|x_{j}\right\|_{j}: N=1,2, \ldots\right\}$ generates the product topology on $\prod_{j=1}^{\infty} X_{j}$ and

$$
\begin{aligned}
\left|\left\langle Z u, L \circ Z^{-1}\right\rangle\right| & =|\langle u, L\rangle|=\left|\left\langle[u]_{l}, L_{l}\right\rangle\right| \leq C\left\|[u]_{l}\right\|_{l} \\
& \leq C\left\|\left([u]_{j}\right)\right\|_{(l)}=C\|Z(u)\|_{(l)}
\end{aligned}
$$


holds for all $u \in B_{p(\cdot)}^{\text {loc }}(\Omega)$. By the Hahn-Banach theorem, $L \circ Z^{-1}$ can be extended to a continuous linear functional on $\prod_{j=1}^{\infty} X_{j}$. Then, by using the isomorphism

$$
A: \bigoplus_{j=1}^{\infty} X_{j}^{\prime} \longrightarrow\left(\prod_{j=1}^{\infty} X_{j}\right)^{\prime},
$$

defined by $\left\langle\left(x_{j}\right), A\left(\left(x_{j}^{\prime}\right)\right)\right\rangle=\sum_{j=1}^{\infty}\left\langle x_{j}, x_{j}^{\prime}\right\rangle$ (see, e.g., [13, p. $284])$, we find $\left(\xi_{j}\right) \in \bigoplus_{j=1}^{\infty} X_{j}^{\prime}$ such that $A\left(\left(\xi_{j}\right)\right)=\left(L \circ Z^{-1}\right)^{-}$ and we obtain the following representation of $L$ :

$$
\langle u, L\rangle=\sum_{j=1}^{\infty}\left\langle[u]_{j}, \xi_{j}\right\rangle, \quad u \in B_{p(\cdot)}^{\mathrm{loc}}(\Omega) .
$$

Now we shall prove that the mapping

$$
\Phi_{p(\cdot)}:\left(B_{p(\cdot)}^{\text {loc }}(\Omega)\right)^{\prime} \longrightarrow B_{\infty}^{c}(\Omega),
$$

defined by $\Phi_{p(\cdot)}(L)=\sum_{j=1}^{\infty}\left[\xi_{j}\right]$, is an algebraic isomorphism (here $\left(\xi_{j}\right)$ is the sequence which represents to $L$ and, for every $j,\left[\xi_{j}\right]$ is the tempered distribution defined by $\left\langle\varphi,\left[\xi_{j}\right]\right\rangle=$ $\left\langle[\varphi]_{j}, \xi_{j}\right\rangle$ for all $\left.\varphi \in S\right)$. Let us see that $\Phi_{p(\cdot)}$ is well defined: (i) First we show that each $\left[\xi_{j}\right] \in B_{\infty}^{c}(\Omega)$. If $\varphi_{\nu} \rightarrow 0$ in $S$ then $\left(\chi_{j} \varphi_{v}\right)^{\wedge} \rightarrow 0$ in $S$ and so in $L_{p(\cdot)}$; therefore $\left\|\left[\varphi_{\nu}\right]_{j}\right\|_{j} \leq$ $\left\|\left[\varphi_{\nu}\right]_{j}\right\|_{p(\cdot), \chi_{j}}^{*}=\left\|\varphi_{\nu}\right\|_{p(\cdot), \chi_{j}}=\left\|\left(\chi_{j} \varphi_{\nu}\right)^{\wedge}\right\|_{p(\cdot)} \rightarrow 0$, that is, $\left[\varphi_{\nu}\right]_{j} \rightarrow$ 0 in $X_{j}$. As a consequence, $\left\langle\varphi_{v},\left[\xi_{j}\right]\right\rangle=\left\langle\left[\varphi_{v}\right]_{j}, \xi_{j}\right\rangle \rightarrow 0$ and $\left[\xi_{j}\right]$ becomes a tempered distribution. Furthermore, for each $\varphi \in C_{0}^{\infty}\left(\mathbb{R}^{n} \backslash K_{j+1}\right)$, we have

$$
\begin{aligned}
\left|\left\langle\varphi,\left[\xi_{j}\right]\right\rangle\right| & =\left|\left\langle[\varphi]_{j}, \xi_{j}\right\rangle\right| \leq\left\|\xi_{j}\right\|\left\|[\varphi]_{j}\right\|_{j} \\
& =\left\|\xi_{j}\right\|\left\|[\varphi]_{j}\right\|_{p(\cdot), \chi_{j}}^{*}=\left\|\xi_{j}\right\|\|\varphi\|_{p(\cdot), \chi_{j}} \\
& =\left\|\xi_{j}\right\|\left\|\chi_{j} \varphi\right\|_{B_{p(\cdot)}}=0
\end{aligned}
$$

(since supp $\chi_{j} \subset \stackrel{\circ}{K}_{j+1}$ ) and so $\operatorname{supp}\left[\xi_{j}\right] \subseteq K_{j+1}$. Thus (see, e.g., $[26$, p. 165$])\left[\xi_{j}\right]^{\wedge}$ coincides with the Fourier-Laplace transform of $\left[\xi_{j}\right]$ defined by

$$
\begin{array}{r}
{\left[\xi_{j}\right]^{\wedge}(x)=\left\langle\chi_{j+2} e^{-i(\cdot) x},\left[\xi_{j}\right]\right\rangle=\left\langle\left[\chi_{j+2} e^{-i(\cdot) x}\right]_{j}, \xi_{j}\right\rangle,} \\
x \in \mathbb{R}^{n} .
\end{array}
$$

Taking here absolute values and using [2, Lemma 3.2.5], we get

$$
\begin{aligned}
& \left|\left[\xi_{j}\right]^{\wedge}(x)\right| \leq\left\|\xi_{j}\right\|\left\|\left[\chi_{j+2} e^{-i(\cdot) x}\right]_{j}\right\|_{j} \leq\left\|\xi_{j}\right\| \\
& \cdot\left\|\chi_{j+2} e^{-i(\cdot) x}\right\|_{p(\cdot), \chi_{j}}=\left\|\xi_{j}\right\|\left\|\chi_{j} e^{-i(\cdot) x}\right\|_{B_{p(\cdot)}}=\left\|\xi_{j}\right\|
\end{aligned}
$$

$$
\begin{aligned}
& \cdot\left\|\left(\chi_{j} e^{-i(\cdot) x}\right)^{\wedge}\right\|_{p(\cdot)}=\left\|\xi_{j}\right\|\left\|\left|\hat{\chi}_{j}(x+(\cdot))\right|^{p_{0}}\right\|_{p^{(\cdot) / p_{0}}}^{1 / p_{0}} \\
& \leq \max \left\{\left(\int_{\mathbb{R}^{n}}\left|\hat{\chi}_{j}(x+y)\right|^{p(y)} d y\right)^{1 / p^{-}},\right. \\
& \left.\left(\int_{\mathbb{R}^{n}}\left|\hat{\chi}_{j}(x+y)\right|^{p(y)} d y\right)^{1 / p^{+}}\right\} \leq 2^{1 / p^{-}-1} \\
& \cdot \max \left\{\left\|\hat{\chi}_{j}\right\|_{p^{-}}+\left\|\hat{\chi}_{j}\right\|_{p^{+}}^{p^{+} / p^{-}},\left\|\hat{\chi}_{j}\right\|_{p^{+}}+\left\|\hat{\chi}_{j}\right\|_{p^{-}}^{p^{-} / p^{+}}\right\}, \\
& x \in \mathbb{R}^{n} .
\end{aligned}
$$

Therefore $\left[\xi_{j}\right]^{\wedge} \in L_{\infty}$ and $\left[\xi_{j}\right] \in B_{\infty}^{c}(\Omega)$. (ii) If $\left(\eta_{j}\right)$ comes from another extension $\left(L \circ Z^{-1}\right)^{=}$, then $\sum_{j=1}^{\infty}\left[\xi_{j}\right]=\sum_{j=1}^{\infty}\left[\eta_{j}\right]$ since

$$
\begin{aligned}
\left\langle\varphi, \sum_{j=1}^{\infty}\left[\xi_{j}\right]\right\rangle & =\sum_{j=1}^{\infty}\left\langle\varphi,\left[\xi_{j}\right]\right\rangle=\sum_{j=1}^{\infty}\left\langle[\varphi]_{j}, \xi_{j}\right\rangle \\
& =\langle\varphi, L\rangle=\sum_{j=1}^{\infty}\left\langle[\varphi]_{j}, \eta_{j}\right\rangle \\
& =\sum_{j=1}^{\infty}\left\langle\varphi,\left[\eta_{j}\right]\right\rangle=\left\langle\varphi, \sum_{j=1}^{\infty}\left[\eta_{j}\right]\right\rangle
\end{aligned}
$$

holds for all $\varphi \in S$.

We have proved that $\Phi_{p(\cdot)}$ is well defined, and it is obvious that it is linear. If $\Phi_{p(\cdot)}(L)=0$ then $\langle\varphi, L\rangle=0$ for all $\varphi \in S$, but $S$ is dense in $B_{p(\cdot)}^{\text {loc }}(\Omega)$ [5, Theorem 3.7/2]; thus $L=0$ and $\Phi_{p(\cdot)}$ is injective. Let us see that $\Phi_{p(\cdot)}$ is surjective: Let $\nu$ be an element of $B_{\infty}^{c}(\Omega)$. We now define the functional

$$
\begin{aligned}
\langle u, L\rangle:=(2 \pi)^{-n} \sum_{j=1}^{\infty} \int_{\mathbb{R}^{n}}\left(\chi_{j} u\right)^{\wedge \sim}\left(\theta_{j} v\right)^{\wedge} d x, & \\
u & \in B_{p(\cdot)}^{\text {loc }}(\Omega),
\end{aligned}
$$

and we show that it is continuous. These integrals converge because $\chi_{j} u \in B_{p(\cdot)} \cap \mathscr{E}^{\prime}\left(K_{j+1}\right)$ that is $\left(\chi_{j} u\right)^{\wedge} \in L_{p(\cdot)}^{-K_{j+1}}$, $\theta_{j} \nu \in B_{\infty} \cap \mathscr{E}^{\prime}\left(K_{j}\right)$ that is $\left(\theta_{j} \nu\right)^{\wedge} \in L_{\infty}^{-K_{j}}$, and $L_{p(\cdot)}^{-K_{j+1}} \hookrightarrow L_{1}^{-K_{j+1}}$ [4, Theorem 3.5]. Moreover, by the properties of the $C_{0}^{\infty}(\Omega)$ partition of unity $\left(\theta_{j}\right)$, there exists a positive integer $m$ such that $\theta_{j} \nu=0$ for all $j>m$ and $\nu=\sum_{j=1}^{m}\left(\theta_{j} \nu\right)$. Then we have that

$$
\begin{aligned}
|\langle u, L\rangle| & \leq C \sum_{j=1}^{m} \int_{\mathbb{R}^{n}}\left|\left(\chi_{j} u\right)^{\wedge \sim}\right|\left|\left(\theta_{j} \nu\right)^{\wedge}\right| d x \\
& \leq C \sum_{j=1}^{m}\left\|\left(\theta_{j} \nu\right)^{\wedge}\right\|_{\infty}\left\|\left(\chi_{j} u\right)^{\wedge}\right\|_{1}
\end{aligned}
$$




$$
\begin{aligned}
& \leq C \sum_{j=1}^{m}\left\|\theta_{j} v\right\|_{B_{\infty}}\left\|\left(\chi_{j} u\right)^{\wedge}\right\|_{p(\cdot)} \\
& =C \sum_{j=1}^{m}\left\|\theta_{j} \nu\right\|_{B_{\infty}}\|u\|_{p(\cdot), \chi_{j}} \\
& \leq\left(C \sum_{j=1}^{m}\left\|\theta_{j} \nu\right\|_{B_{\infty}}\right)\|u\|_{p(\cdot), \chi_{m}}
\end{aligned}
$$

holds for all $u \in B_{p(\cdot)}^{\text {loc }}(\Omega)$ and so $L \in\left(B_{p(\cdot)}^{\text {loc }}(\Omega)\right)^{\prime}$. Finally we check that $\Phi_{p(\cdot)}(L)=v$ : Assume that $\left(\xi_{j}\right) \in \bigoplus_{j=1}^{\infty} X_{j}^{\prime}$ represents to $L$ and recall that $\chi_{j} \theta_{j}=\theta_{j}(j=1,2, \ldots)$ and $(\widehat{\psi})^{\wedge}=(2 \pi)^{n} \widetilde{\psi}$ (for all $\left.\psi \in S\right)$; then

$$
\begin{aligned}
\left\langle\varphi, \Phi_{p(\cdot)}(L)\right\rangle & =\left\langle\varphi, \sum_{j=1}^{\infty}\left[\xi_{j}\right]\right\rangle=\sum_{j=1}^{\infty}\left\langle\varphi,\left[\xi_{j}\right]\right\rangle \\
& =\sum_{j=1}^{\infty}\left\langle[\varphi]_{j}, \xi_{j}\right\rangle=\langle\varphi, L\rangle \\
& =(2 \pi)^{-n} \sum_{j=1}^{m} \int_{\mathbb{R}^{n}}\left(\chi_{j} \varphi\right)^{\wedge \sim}\left(\theta_{j} \nu\right)^{\wedge} d x \\
& =(2 \pi)^{-n} \sum_{j=1}^{m}\left\langle\left(\chi_{j} \varphi\right)^{\wedge \sim},\left(\theta_{j} \nu\right)^{\wedge}\right\rangle \\
& =\sum_{j=1}^{m}\left\langle\chi_{j} \varphi, \theta_{j} \nu\right\rangle=\sum_{j=1}^{m}\left\langle\chi_{j} \theta_{j} \varphi, \nu\right\rangle \\
& =\sum_{j=1}^{m}\left\langle\theta_{j} \varphi, \nu\right\rangle=\left\langle\varphi, \sum_{j=1}^{m} \theta_{j} \nu\right\rangle \\
& =\langle\varphi, \nu\rangle
\end{aligned}
$$

holds for all $\varphi \in S$.

Finally, if $p(\cdot) \equiv p$ and $0<p \leq 1$ then the HardyLittlewood maximal operator $M$ is bounded on $L_{p / p_{0}}$ for each $\left.p_{0} \in\right] 0, p\left[\right.$ and so we also have that $\left(B_{p}^{\text {loc }}(\Omega)\right)^{\prime}$ is algebraically isomorphic to $B_{\infty}^{c}(\Omega)$.

Now we prove a result we use to calculate the Fréchet envelope of $B_{p(\cdot)}^{\text {loc }}(\Omega)$.

Theorem 7. Let $X[\mathscr{T}]$ be an F-space such that its dual $X^{\prime}:=(X[\mathscr{T}])^{\prime}$ separates points of $X$. Assume that $X$ is a dense linear subspace of a Fréchet space $Y[\mathcal{S}]$, that the inclusion map $\iota: X[\mathscr{T}] \hookrightarrow Y[\mathcal{S}]$ is continuous, and that $Y^{\prime}=X^{\prime}\left(Y^{\prime}:=(Y[\delta])^{\prime}\right)$, that is, the transpose of $l$, ${ }^{t} l$, is an algebraic isomorphism. Assume finally that $Z$ is a complemented subspace of $X$. Then, we have the following:

(1) $Y[\mathcal{S}]=\widehat{X}$; that is, $Y[\mathcal{S}]$ is the Fréchet envelope of $X[\mathscr{T}], \bar{Z}^{Y[\mathcal{S}]}=\widehat{Z}$, and $\widehat{Z}$ is also a complemented subspace of $Y[\mathcal{S}]$.
(2) If furthermore $Y[\mathcal{S}]$ is separable then ${ }^{t} \iota$ becomes an isomorphism of $Y^{\prime}\left[\beta\left(Y^{\prime}, Y\right)\right]$ onto $X^{\prime}[\mathfrak{I}]$ being $\mathfrak{I}$ the topology of the uniform convergence on the $m\left(X, X^{\prime}\right)$ bounded subsets of $X$.

Proof. (1) To see that $\bar{Z}^{Y[\delta]}=\widehat{Z}$ it suffices to show that the induced topology by $\mathcal{S}$ on $Z, \mathcal{S}_{Z}$, coincides with the Mackey topology $m\left(Z, Z^{\prime}\right)(Z$ is also an $F$-space with separating dual since it is a complemented subspace of $X)$. To do so first we observe that $\left(Z\left[\mathcal{S}_{Z}\right]\right)^{\prime}=Z^{\prime}\left(:=\left(Z\left[\mathscr{T}_{Z}\right]\right)^{\prime}\right)$ (obviously $\left(Z\left[\mathcal{S}_{Z}\right]\right)^{\prime} \subset Z^{\prime}$ and, on the other hand, if $z^{\prime} \in Z^{\prime}$ and $x^{\prime} \in X^{\prime}$ is an extension of $z^{\prime}$ then this $x^{\prime}$ has the form $x^{\prime}=y^{\prime} \circ \iota$, with $y^{\prime} \in Y^{\prime}$; thus $\left.z^{\prime} \in\left(Z\left[\mathcal{S}_{Z}\right]\right)^{\prime}\right)$ and then recall that every metrizable locally convex space has the Mackey topology (see, e.g., $[13$, pp. 379,380$])$. In particular, we have also shown that $Y[\delta]=\widehat{X}$.

It remains to prove the last claim. Let $P$ be a continuous projection in $X[\mathscr{T}]$ such that $\operatorname{Im} P=Z$ and let $\widehat{P}: Y[\mathcal{S}] \rightarrow$ $Y[\mathcal{S}]$ be the unique extension linear and continuous of $P$ (recall that $Y[\mathcal{S}]$ is $\widehat{X}$ ). Since $\widehat{Z}=\bar{Z}^{Y[\mathcal{S}]}, \widehat{P}$ is also a linear and continuous mapping from $Y[\mathcal{S}]$ into $\bar{Z}^{Y[\mathcal{S}]}$. Furthermore, if $y \in \bar{Z}^{Y[\mathcal{S}]}$ and $\left(z_{n}\right)$ is a sequence in $Z$ convergent to $y$ in $Y[\mathcal{S}]$ then $\widehat{P}(y)=\lim _{n} \widehat{P}\left(z_{n}\right)=\lim _{n} P\left(z_{n}\right)=\lim _{n} z_{n}=y$. Therefore, $\operatorname{Im} \widehat{P}=\bar{Z}^{Y[\mathcal{S}]}$. To conclude we check that $\widehat{P}$ is a projection: If $y \in Y$ and $\left(x_{n}\right)$ is a sequence in $X$ such that $\lim _{n} x_{n}=y$ in $Y[\mathcal{S}]$ then $\widehat{P}(y)=\lim _{n} P\left(x_{n}\right)$ in $Y[\mathcal{S}]$ and so $\widehat{P}^{2}(y)=\widehat{P}\left(\lim _{n} P\left(x_{n}\right)\right)=\lim _{n} \widehat{P}\left(P\left(x_{n}\right)\right)=\lim _{n} P^{2}\left(x_{n}\right)=$ $\lim _{n} P\left(x_{n}\right)=\widehat{P}(y)$.

(2) The continuity of the mapping ${ }^{t} \iota$ follows from the fact that every $m\left(X, X^{\prime}\right)$-bounded subset of $X$ is $\mathcal{S}$-bounded subset of $Y$ (by (1), $\mathcal{\delta}_{X}=m\left(X, X^{\prime}\right)$ ). If $Y[\delta]$ is separable, every bounded subset of the Fréchet space $Y[\mathcal{S}]$ is contained in the closure of a bounded subset of $X\left[\mathcal{S}_{X}\right]$ (apply $[13,(1) \mathrm{p}$. 403]), and since $\mathcal{S}_{X}=m\left(X, X^{\prime}\right)$, it follows that the mapping $\left({ }^{t} \iota\right)^{-1}$ is also continuous.

Remark 8. (1) In [21] Shapiro constructs subspaces of the $F$-space (of harmonic functions) $h_{p^{-}}$isomorphic to $l_{\infty}$ and also proves that the Fréchet envelope of $h_{p^{-}}$is the separable Fréchet space $b_{p^{-}}$(see notations in [21]). From Theorem 7 it follows that these subspaces are not complemented in $h_{p^{-}}$.

(2) If in Theorem $7 X[\mathscr{T}]=\left(X,\|\cdot\|_{X}\right)$ is a quasi-Banach space and $Y[\delta]=\left(Y,\|\cdot\|_{Y}\right)$ is a Banach space not necessarily separable, then $Y[\mathcal{S}]$ is the Banach envelope of $X[\mathscr{T}]$ and ${ }^{t} t$ is an isomorphism of $Y^{\prime}$ onto $X^{\prime}$ (these spaces equipped with the norms $\left\|y^{\prime}\right\|=\sup _{\|y\|_{Y} \leq 1}\left|\left\langle y, y^{\prime}\right\rangle\right|,\left\|x^{\prime}\right\|=\sup _{\|x\|_{X} \leq 1}\left|\left\langle x, x^{\prime}\right\rangle\right|$, resp.): It suffices to take into account that if $E$ is a Banach space and $F$ a dense linear subspace; then every bounded subset of $E$ is contained in the closure of a bounded subset of $F$.

Thus Theorem 7 recovers known results (see, e.g., [18, Theorem 5]).

In Proposition 9 and Theorem 10 we will use the same notation as in the proof of Theorem 6 . 
Proposition 9. Let $\mathfrak{I}_{1}$ be the topology on $\left(B_{p(\cdot)}^{\text {loc }}(\Omega)\right)^{\prime}$ of the uniform convergence on the bounded subsets of $B_{p(\cdot)}^{\text {loc }}(\Omega)$. Then the mapping $\Phi_{p(\cdot)}:\left(B_{p(\cdot)}^{\text {loc }}(\Omega)\right)^{\prime}\left[\mathfrak{I}_{1}\right] \rightarrow B_{\infty}^{c}(\Omega)$ is open. If $p(\cdot) \equiv 1$ then $\Phi_{1}$ becomes an isomorphism.

Proof. First we show that $\Phi_{p(\cdot)}^{-1}$ is continuous $\left(\Leftrightarrow \Phi_{p(\cdot)}\right.$ is open). For this it suffices to check, since $B_{\infty}^{c}(\Omega)=\operatorname{ind}_{j}\left[B_{\infty} \cap\right.$ $\left.\mathscr{E}^{\prime}\left(K_{j}\right)\right]$, that, for every $j, \Phi_{p(\cdot)}^{-1}$ is continuous from $B_{\infty} \cap$ $\mathscr{E}^{\prime}\left(K_{j}\right)$ into $\left(B_{p(\cdot)}^{\text {loc }}(\Omega)\right)^{\prime}\left[\mathfrak{I}_{1}\right]$. Fix $j$ and let $m$ be a positive integer such that, for all $v \in B_{\infty} \cap \mathscr{E}^{\prime}\left(K_{j}\right), \theta_{l} \nu=0$ for all $l>m$ and $v=\sum_{l=1}^{m}\left(\theta_{l} \nu\right)$. Let $M$ be a bounded subset of $B_{p(\cdot)}^{\text {loc }}(\Omega)$; then $\sup _{u \in M}\|u\|_{p(\cdot), \chi_{m}}<\infty$. Now we argue as in the proof of Theorem 6 and we obtain that

$$
\begin{aligned}
& \sup _{u \in M}\left|\left\langle u, \Phi_{p(\cdot)}^{-1}(v)\right\rangle\right| \leq C \sum_{l=1}^{m}\left\|\left(\theta_{l} \nu\right)^{\wedge}\right\|_{\infty} \sup _{u \in M}\|u\|_{p(\cdot), \chi_{m}} \\
& =C \sum_{l=1}^{m}\left\|\hat{\theta}_{l} * \hat{v}\right\|_{\infty} \sup _{u \in M}\|u\|_{p(\cdot), \chi_{m}} \\
& \leq\left(C \sum_{l=1}^{m}\left\|\hat{\theta}_{l}\right\|_{1} \sup _{u \in M}\|u\|_{p(\cdot), \chi_{m}}\right)\|v\|_{B_{\infty}}
\end{aligned}
$$

holds for all $v \in B_{\infty} \cap \mathscr{E}^{\prime}\left(K_{j}\right)$, which shows the continuity of $\Phi_{p(\cdot)}^{-1}$. If $p(\cdot) \equiv 1$, then $\mathfrak{I}_{1}$ is the strong topology $\beta\left(\left(B_{1}^{\text {loc }}(\Omega)\right)^{\prime}, B_{1}^{\text {loc }}(\Omega)\right)$. By a result of Vogt $[10] B_{1}^{\text {loc }} \simeq\left(l_{1}\right)^{\mathbb{N}}$, and thus $\left(B_{1}^{\text {loc }}(\Omega)\right)^{\prime}\left[\mathfrak{I}_{1}\right] \simeq\left(l_{\infty}\right)^{(\mathbb{N})}$ (apply, e.g., [13, p. 287]). Hence it follows that $\left(B_{1}^{\text {loc }}(\Omega)\right)^{\prime}\left[\mathfrak{I}_{1}\right]$ is an $(L B)$-space. Since $B_{\infty}^{c}(\Omega)$ is also an $(L B)$-space, we can apply [14, (4) b p. 43] to $\Phi_{1}^{-1}$ and conclude that $\Phi_{1}$ is an isomorphism.

The next theorem improves the first part of the previous result considering the topology of the uniform convergence on the $m\left(B_{p(\cdot)}^{\text {loc }}(\Omega),\left(B_{p(\cdot)}^{\text {loc }}(\Omega)\right)^{\prime}\right)$-bounded subsets of $B_{p(\cdot)}^{\text {loc }}(\Omega)$ instead of the topology $\mathfrak{I}_{1}$. Our method requires the calculation of the Fréchet envelope of $B_{p(\cdot)}^{\text {loc }}(\Omega)$.

Theorem 10. (1) $\overline{B_{p(\cdot)}^{\text {loc }}(\Omega)}=B_{1}^{\text {loc }}(\Omega)$; that is, $B_{1}^{\text {loc }}(\Omega)$ is the Fréchet envelope of $B_{p(\cdot)}^{\mathrm{loc}}(\Omega)$ (in particular, $\widehat{B_{p}^{\mathrm{loc}}(\Omega)}=B_{1}^{\mathrm{loc}}(\Omega)$ for all $0<p \leq 1)$.

(2) If $\mathfrak{T}$ is the topology of the uniform convergence on the $m\left(B_{p(\cdot)}^{\text {loc }}(\Omega),\left(B_{p(\cdot)}^{\text {loc }}(\Omega)\right)^{\prime}\right)$-bounded subsets of $B_{p(\cdot)}^{\text {loc }}(\Omega)$, then the spaces $\left(B_{p(\cdot)}^{\text {loc }}(\Omega)\right)^{\prime}[\mathfrak{I}]$ and $B_{\infty}^{c}(\Omega)$ are isomorphic (in particular, $\left(B_{p}^{\text {loc }}(\Omega)\right)^{\prime}[\mathfrak{T}]$ and $B_{\infty}^{c}(\Omega)$ are isomorphic for all $0<p \leq 1)$.

Proof. (1) $B_{p(\cdot)}^{\text {loc }}(\Omega)$ is an $F$-space on which $\left(B_{p(\cdot)}^{\text {loc }}(\Omega)\right)^{\prime}$ separates points (see Theorem 6 ). Furthermore, $B_{p(\cdot)}^{\text {loc }}(\Omega)$ is a dense linear subspace of the Fréchet space $B_{1}^{\text {loc }}(\Omega)$ [5, Theorem 3.7] and the inclusion map $\iota: B_{p(\cdot)}^{\text {loc }}(\Omega) \hookrightarrow B_{1}^{\text {loc }}(\Omega)$ is continuous (for each $j$ and each $u \in B_{p(\cdot)}^{\text {loc }}(\Omega)$, we have $\|u\|_{1, \chi_{j}}=\left\|\left(\chi_{j} u\right)^{\wedge}\right\|_{1}$ $\leq C\left\|\left(\chi_{j} u\right)^{\wedge}\right\|_{p(\cdot)}=C\|u\|_{p(\cdot), \chi_{j}}$ in virtue of the embedding
$L_{p(\cdot)}^{-K_{j+1}} \hookrightarrow L_{1}^{-K_{j+1}}[4$, Theorem 3.5]). Now we shall see that the following diagram

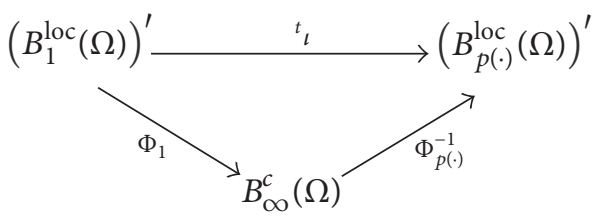

is commutative. Let $L \in\left(B_{1}^{\text {loc }}(\Omega)\right)^{\prime}$. Then $\Phi_{1}(L) \in B_{\infty}^{c}(\Omega)$ and we can find a positive integer $k$ such that $\theta_{j} \Phi_{1}(L) \stackrel{\infty}{=} 0$ for all $j>k$ and $\Phi_{1}(L)=\sum_{j=1}^{k}\left(\theta_{j} \Phi_{1}(L)\right)$ and so (reasoning as in Theorem 6) we have that

$$
\begin{aligned}
\langle\varphi, & \left.\Phi_{p(\cdot)}^{-1}\left(\Phi_{1}(L)\right)\right\rangle \\
= & (2 \pi)^{-n} \sum_{j=1}^{\infty} \int_{\mathbb{R}^{n}}\left(\chi_{j} \varphi\right)^{\wedge \sim}\left(\theta_{j} \Phi_{1}(L)\right)^{\wedge} d x \\
= & (2 \pi)^{-n} \sum_{j=1}^{k}\left\langle\left(\chi_{j} \varphi\right)^{\wedge \sim},\left(\theta_{j} \Phi_{1}(L)\right)^{\wedge}\right\rangle \\
= & \sum_{j=1}^{k}\left\langle\chi_{j} \varphi, \theta_{j} \Phi_{1}(L)\right\rangle=\left\langle\varphi, \Phi_{1}(L)\right\rangle=\langle\varphi, L\rangle \\
= & \left\langle\varphi,{ }^{t} \iota(L)\right\rangle,
\end{aligned}
$$

for all $\varphi \in C_{0}^{\infty}(\Omega)$. Since $C_{0}^{\infty}(\Omega)$ is dense in $B_{p(\cdot)}^{\text {loc }}(\Omega)$, it follows that the previous diagram is commutative and that ${ }^{t} t$ is an algebraic isomorphism. Then, using Theorem 7(1), we conclude that $\left.B_{p(\cdot)}^{\text {loc }(\Omega)}\right)=B_{1}^{\text {loc }}(\Omega)$ and that $\left(B_{p(\cdot)}^{\text {loc }}(\Omega)\right)^{\prime}[\mathfrak{T}]$ and $\left(B_{1}^{\text {loc }}(\Omega)\right)^{\prime}$ are isomorphic via the map ${ }^{t} \iota$.

(2) It is an immediate consequence of (1) and of Proposition 9.

Corollary 11. Let $X$ be a complemented subspace of $B_{p(\cdot)}^{\text {loc }}(\Omega)$. Then $\widehat{X}$ is finite-dimensional or isomorphic to one of the spaces $l_{1}, l_{1}^{\mathbb{N}}, \omega, \omega \times l_{1}$. If furthermore $X$ is locally convex (resp., a quasiBanach space) then $X$ is finite-dimensional or isomorphic to one of the spaces $l_{1}, l_{1}^{\mathbb{N}}, \omega, \omega \times l_{1}$ (resp., $\widehat{X}$ is finite-dimensional or isomorphic to $l_{1}$ ).

Proof. By Theorem 10(1) and Theorem 7(1), $\widehat{X}$ is also a complemented subspace of $B_{1}^{\text {loc }}(\Omega)$. Then, since $B_{1}^{\text {loc }}(\Omega) \simeq$ $l_{1}^{\mathbb{N}}$ (see [10]), $\widehat{X}$ becomes isomorphic to a complemented subspace of $l_{1}^{\mathbb{N}}$. The proof of the first claim concludes by applying Theorem 1.2 of [27]. If $X$ is locally convex then $X$ is a Fréchet or Banach space (see (3), Section 1) and so $X=\widehat{X}$. Finally, if $X$ is a quasi-Banach space, its Banach envelope must necessarily be finite-dimensional or isomorphic to $l_{1}$.

Corollary 12. Let $X$ be an infinite dimensional complemented subspace of $\left(B_{p(\cdot)}^{\text {loc }}(\Omega)\right)^{\prime}[\mathfrak{I}]$. Then $X$ is isomorphic to one of the spaces $l_{\infty},\left(l_{\infty}\right)^{(\mathbb{N})}, \varphi$, or $\varphi \times l_{\infty}$. 
Proof. By Theorem 10(2) $X$ is isomorphic to a complemented subspace of $B_{\infty}^{c}(\Omega)$ and since

$$
B_{\infty}^{c}(\Omega) \simeq\left(B_{1}^{\text {loc }}(\Omega)\right)^{\prime} \simeq\left(l_{\infty}\right)^{(\mathbb{N})}
$$

(see the proof of Proposition 9) $X$ is also isomorphic to a complemented subspace of $\left(l_{\infty}\right)^{(\mathbb{N})}$. The proof concludes by applying [27, Theorem 2.1].

\section{Questions}

(1) To obtain the dual of the space $B_{p(\cdot)}^{\text {loc }}(\Omega)$ when the variable exponent $p(\cdot) \in \mathscr{P}^{0}, p^{-} \leq 1<p^{+}$, and the Hardy-Littlewood maximal operator $M$ is bounded in $L_{p(\cdot) / p_{0}}$ for some $0<p_{0}<p^{-}$.

(2) To obtain a sequence space representation of the space $B_{p(\cdot)}^{\text {loc }}(\Omega)\left(p(\cdot) \in \mathscr{P}^{0}\right)$.

(3) To prove that $B_{p}^{\text {loc }}(\Omega) \simeq l_{p}^{\mathbb{N}}$ for all $0<p<1$. (In a forthcoming paper the authors have shown this isomorphism for $\Omega=\mathbb{R}^{n}$.)

\section{Competing Interests}

The authors declare that there is no conflict of interests regarding the publication of this paper.

\section{Acknowledgments}

The first author has been partially supported by the Spanish Government Grant MTM2014-53009-P.

\section{References}

[1] D. Cruz-Uribe and A. Fiorenza, Variable Lebesgue Spaces, Foundations and Harmonic Analysis, Birkhäuser, Springer, Basel, Switzerland, 2013.

[2] L. Diening, P. Harjulehto, P. Hästö, and M. Růžička, Lebesgue and Sobolev Spaces with Variable Exponents, Lecture Notes in Mathematics 2007, Springer, Berlin, Germany, 2011.

[3] J. Motos, M. J. Planells, and C. F. Talavera, "Duals of variable exponent Hörmander spaces $\left(0<p^{-} \leq p^{+} \leq 1\right)$ and some applications," Revista de la Real Academia de Ciencias Exactas, Físicas y Naturales. Serie A: Matemáticas, vol. 109, no. 2, pp. 657668, 2015.

[4] J. Motos, M. J. Planells, and C. F. Talavera, "On variable exponent Lebesgue spaces of entire analytic functions," Journal of Mathematical Analysis and Applications, vol. 388, no. 2, pp. 775-787, 2012.

[5] J. Motos, M. J. Planells, and C. F. Talavera, "A note on variable exponent Hörmander spaces," Mediterranean Journal of Mathematics, vol. 10, no. 3, pp. 1419-1434, 2013.

[6] L. Hörmander, The Analysis of Linear Partial Operators II, vol. 257, Springer, Berlin, Germany, 1983.

[7] J. Motos and M. J. Planells, "On sequence space representations of Hörmander-Beurling spaces," Journal of Mathematical Analysis and Applications, vol. 348, no. 1, pp. 395-403, 2008.
[8] J. Motos, M. J. Planells, and J. Villegas, "Some embedding theorems for Hörmander-Beurling spaces," Journal of Mathematical Analysis and Applications, vol. 364, no. 2, pp. 473-482, 2010.

[9] M. J. Planells and J. Villegas, "A note on traces of Hörmander spaces," Boletín de la Sociedad Matemática Mexicana (3), vol. 14, no. 1, pp. 85-94, 2008.

[10] D. Vogt, "Sequence space representations of spaces of test functions and distributions," in Functional Analysis, Holomorphy and Approximation Theory, G. I. Zapata, Ed., vol. 83 of Lecture Notes in Pure and Applied Mathematics, pp. 405-443, Dekker, New York, NY, USA, 1983.

[11] H. Jarchow, Locally Convex Spaces, Teubner, Stuttgart, Germany, 1981.

[12] N. J. Kalton, N. T. Peck, and J. W. Roberts, An F-space sampler, vol. 89 of London Mathematical Society Lecture Note Series, Cambridge University Press, Cambridge, Uk, 1985.

[13] G. Köthe, Topological Vector Spaces I, vol. 159, Springer, Berlin, Germany, 1969.

[14] G. Köthe, Topological Vector Spaces II, vol. 237, Springer, Berlin, Germany, 1979.

[15] N. J. Kalton, "Quasi-Banach spaces," in Handbook of the Geometry of Banach Spaces, W. B. Johnson and J. Lindenstrauss, Eds., vol. 2, pp. 1099-1130, Elsevier, Amsterdam, The Netherlands, 2003.

[16] D. Cruz-Uribe, A. Fiorenza, J. M. Martell, and C. Pérez, "The boundedness of classical operators on variable $L^{p}$ spaces," Annales Academiae Scientiarum Fennicae Mathematica, vol. 31, no. 1, pp. 239-264, 2006.

[17] H. Triebel, Theory of Function Spaces, vol. 78, Birkhäuser, Basel, Switzerland, 1983.

[18] O. Mendez and M. Mitrea, "The Banach envelopes of Besov and Triebel-Lizorkin spaces and applications to partial differential equations," Journal of Fourier Analysis and Applications, vol. 6, no. 5, pp. 503-531, 2000.

[19] M. Hoffmann, "The Banach envelope of Paley-Wiener type spaces," Proceedings of the American Mathematical Society, vol. 131, no. 2, pp. 543-548, 2003.

[20] J. H. Shapiro, "Mackey topologies, reproducing kernels, and diagonal maps on the Hardy and Bergman spaces," Duke Mathematical Journal, vol. 43, no. 1, pp. 187-202, 1976.

[21] J. H. Shapiro, "Some F-spaces of harmonic functions for which the Orlicz-Pettis theorem fails," Proceedings London Mathematical Society, vol. 3, no. 50, pp. 299-313, 1985.

[22] J. C. Díaz, "A note on isomorphisms between powers of Banach spaces," Collectanea Mathematica, vol. 38, no. 2, pp. 137-140, 1987.

[23] R. P. Boas, Entire Functions, Academic Press, 1954.

[24] S. Boza, Espacios de Hardy discretos y acotación de operadores [thesis], Universitat de Barcelona, Barcelona, Spain, 1998.

[25] H. Triebel, Fourier Analysis and Function Spaces, vol. 7 of Texts in Mathematics, Teubner, Leipzig, Germany, 1977.

[26] L. Hörmander, The Analysis of Linear Partial Operators I, vol. 256 of Grundlehren, Springer, Berlin, Germany, 1983.

[27] G. Metafune and V. B. Moscatelli, "Complemented subspaces of sums and products of Banach spaces," Annali di Matematica Pura ed Applicata, vol. 153, no. 4, pp. 175-190, 1989. 


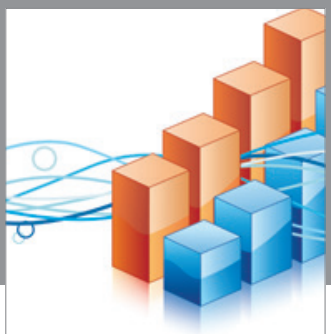

Advances in

Operations Research

vatem alat4

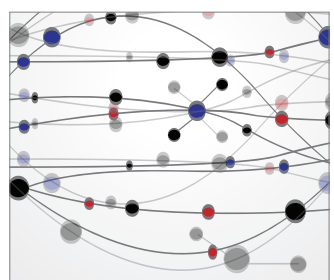

\section{The Scientific} World Journal
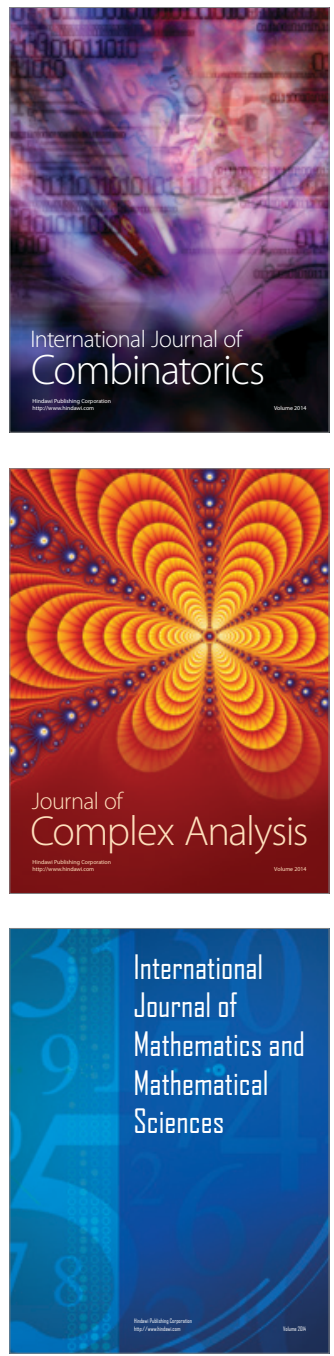
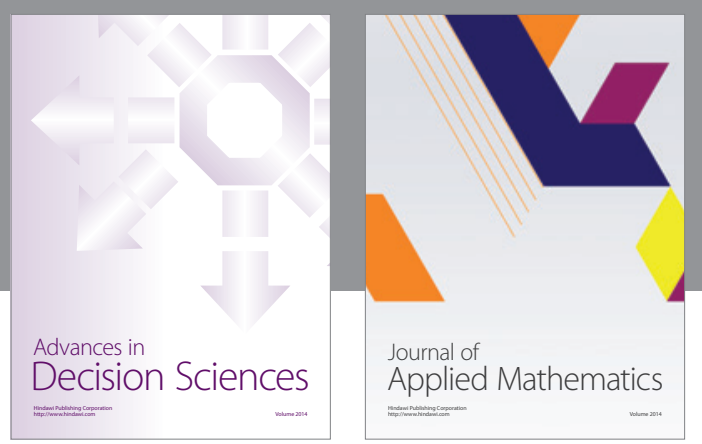

Algebra

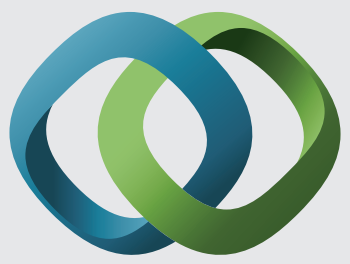

\section{Hindawi}

Submit your manuscripts at

http://www.hindawi.com
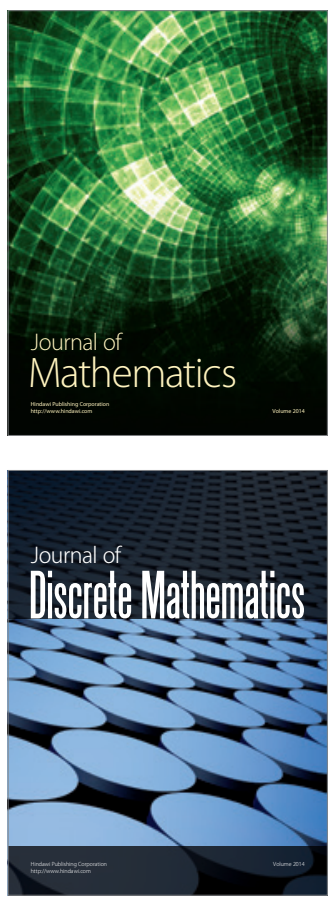

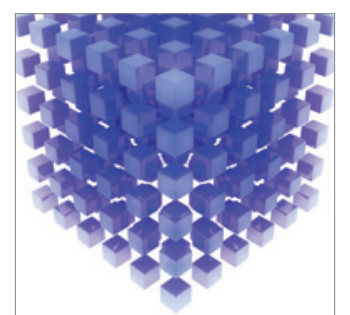

Mathematical Problems in Engineering
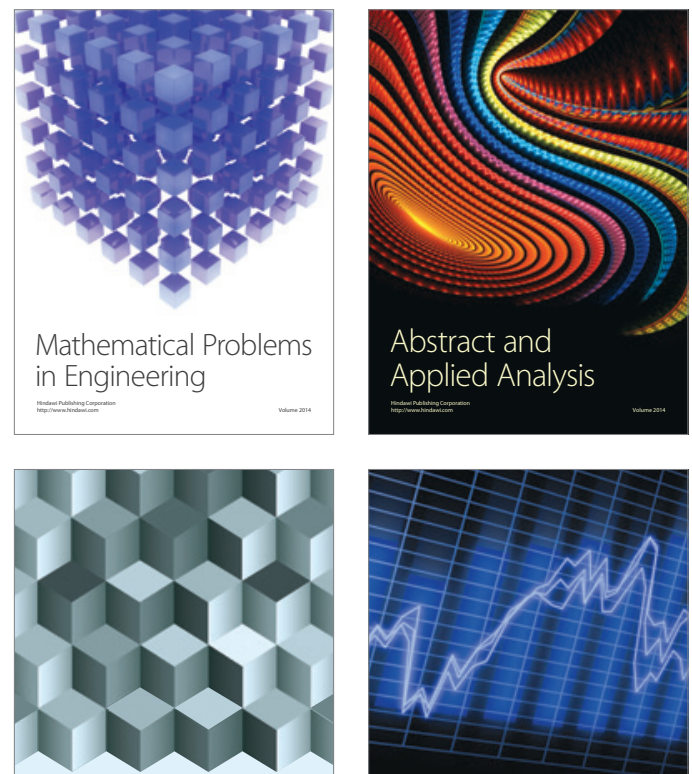

Journal of

Function Spaces

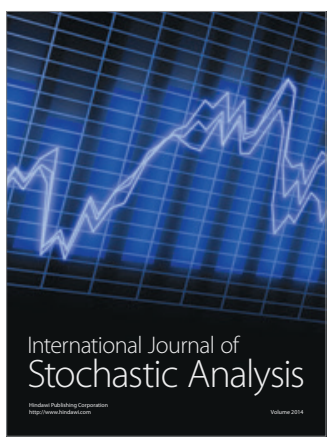

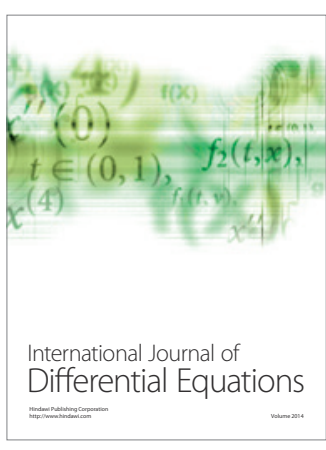
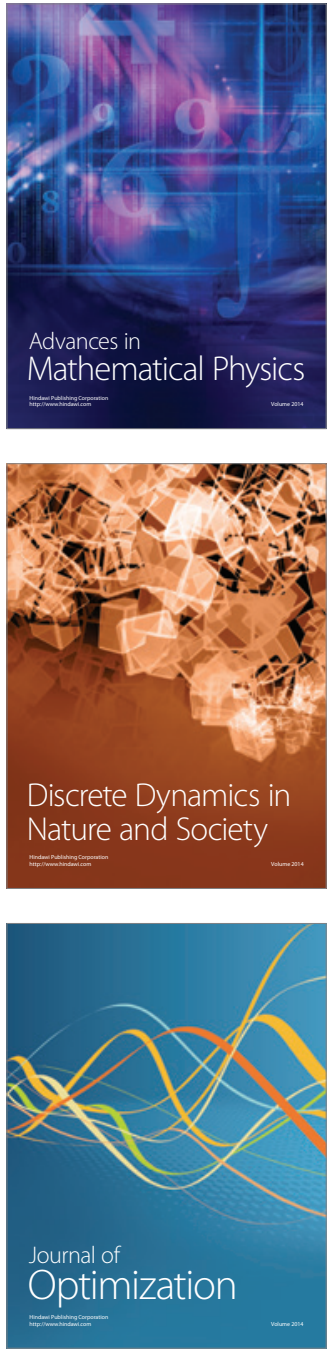